\title{
IRBFN-based multiscale solution of a model 1D elliptic equation
}

\author{
D.-A. An-Vo, C.-D. Tran, N. Mai-Duy \& T. Tran-Cong \\ Computational Engineering and Science Research Centre, \\ University of Southern Queensland, Australia
}

\begin{abstract}
Many engineering problems have a wide range of length scales in their solutions. Direct numerical simulations for these problems typically require extremely-large amounts of CPU time and computer memory, which may be too expensive or impossible on the present supercomputers. In this paper, we present a high-order method, based on the multiscale basis function framework and integrated radialbasis-function networks, for solving multiscale elliptic problems in one dimension. Keywords: integrated radial basis functions, point collocation, subregion collocation, multiscale elliptic problems.
\end{abstract}

\section{Introduction}

In composite materials, the presence of particles/fibres in the resin gives rise to the multiscale fluctuations in the thermal or electrical conductivity. In porous media, formation properties such as permeability have a very high degree of spatial variability. These effects are typically captured at scales that are too fine for direct numerical simulation. To enable the solution of these problems, a number of advanced numerical methods have been developed. Examples include those based on the homogenisation theory (e.g. [1]), upscaling methods (e.g. [2]) and multiscale methods (e.g. [3]). The homogenisation-theory-based methods have been successfully applied for the prediction of effective properties and statistical correlation lengths for multicomponent random media. However, restrictive assumptions on the media, such as scale separation and periodicity, limit their range of application. Furthermore, when dealing with problems having many separate scales, they become very expensive because their computational cost increases exponentially with the number of scales. For upscaling methods, their design 
principle is based on simple physical and/or mathematical motivations. A heterogeneous medium is replaced by a homogeneous medium characterised by equivalent properties, and coarse-scale equations are prescribed in explicit form. Although upscaling techniques are effective, most of their applications have been reported for the case of periodic structures. As opposed to upscaling, multiscale methods consider the full problem with the original resolution. The coarsescale equations are formed and solved numerically, where one constructs the basis functions from the leading order homogeneous elliptic equation in coarsescale elements. The idea of using the non-polynomial multiscale approximation space rather than the standard piecewise polynomial space was first introduced by Babuška et al. [4] for one-dimensional problems and by Hou and $\mathrm{Wu}$ [3] for two-dimensional elliptic problems. These methods have the ability to capture accurately the effects of fine-scale variations without the need for using global fine meshes. Multiscale methods can be categorised into multiscale finite-element (FE) methods (e.g. [5, 6]), mixed multiscale FE methods (e.g. [7, 8]) and multiscale control-volume (CV) methods (e.g. $[9,10])$. Typically, there are two different meshes used: a fine mesh for computing locally the basis function space, and a coarse mesh for computing globally the solution of an elliptic partial differential equation (PDE). The multiscale bases are independent of each other and their constructions can thus be conducted in parallel. In solving the elliptic PDE, one may only need to employ a mesh that today's computing resources can afford for computing. For two-scale periodic structures, Hou et al. [11] have proved that the multiscale FE method indeed converges to the correct solution independent of the small scale in the homogenisation limit. Multiscale techniques require the solutions of elliptic PDEs which are achieved by means of discretisation schemes.

Radial-basis-function networks (RBFNs) are known as a powerful tool for the approximation of scattered data. Their application to the solution of PDEs has received a great deal of attention over the last 20 years (e.g. [12] and references therein). It is easy to implement RBF collocation methods and they can give a high-order convergence solution. A number of RBF approaches, based on local approximations, domain decompositions, preconditioning schemes and compactly-supported basis functions, have been presented, towards the solution of large-scale problems. Integrated RBFNs (IRBFNs) proposed by Mai-Duy and Tran-Cong [13, 14], in which highest-order derivatives under consideration are approximated using RBFNs and lower-order derivatives are obtained through integration, have several advantages over conventional differentiated RBFNs. The purposes of using integration (a smoothing operator) to construct the approximants are (i) to avoid the reduction in convergence rate caused by differentiation and (ii) to improve the numerical stability of a discrete solution.

This paper is concerned with the incorporation of IRBFNs and subregion collocation (i.e. control-volume (CV) formulation) into the non-polynomial approximation space approach, called msIRBFN-CV method, for solving onedimensional multiscale elliptic problems. The remainder of the paper is organised as follows. Section 2 presents the governing equation. The proposed method is 
described in Section 3 and numerical results are given in Section 4. Section 5 concludes the paper.

\section{Governing equation}

A class of multiscale problems arising from composite material and porous media can be modelled by second-order elliptic PDEs

$$
-\nabla \cdot\left(a^{\varepsilon} \nabla u\right)=f
$$

where the coefficient $a^{\varepsilon}$ is an oscillatory function involving a small scale. In the case of heat conduction in composite materials, $u$ and $a$ represent the temperature and thermal conductivity, respectively. In the case of flows in porous media, $u$ is the pressure and $a=k / \nu$ is the ratio of the permeability $k$ and the fluid viscosity $\nu$. In this work, we consider (1) in one dimension.

Conventional discretisation techniques using piecewise polynomial approximation spaces can be applied to solve (1). However, it would require the mesh size $h$ be much smaller than the finest scale, i.e., $h \ll \varepsilon$. In contrast, the multiscale computational framework uses a coarse grid of size $h>\varepsilon$ and an adaptive basis which incorporates the small-scale features of the oscillating coefficient function $a^{\varepsilon}(\mathbf{x})$. Integrated RBFNs are designed to construct the multiscale basis function space.

\section{Present msIRBFN-CV method}

\subsection{Integrated radial-basis-function networks}

IRBFNs, which are employed with the multiquadric function, for the representation of a function $y$ and its derivatives (e.g. up to the second order) in one dimension can be mathematically described as

$$
\begin{aligned}
\frac{d^{2} y}{d x^{2}}(x) & =\sum_{i=1}^{m} w_{i} G_{i}(x) \\
\frac{d y}{d x}(x) & =\sum_{i=1}^{m} w_{i} H_{i}(x)+C_{1} \\
y(x) & =\sum_{i=1}^{m} w_{i} \bar{H}_{i}(x)+C_{1} x+C_{2}
\end{aligned}
$$

where $C_{1}$ and $C_{2}$ are constants of integration and

$$
G_{i}(x)=\left(\left(x-c_{i}\right)^{2}+a_{i}^{2}\right)^{1 / 2}
$$




$$
\begin{aligned}
H_{i}(x) & =\int G_{i}(x) d x=\frac{\left(x-c_{i}\right)\left(\left(x-c_{i}\right)^{2}+a_{i}^{2}\right)^{1 / 2}}{2} \\
& +\frac{a_{i}^{2}}{2} \ln \left(\left(x-c_{i}\right)+\left(\left(x-c_{i}\right)^{2}+a_{i}^{2}\right)^{1 / 2}\right) \\
\bar{H}_{i}(x) & =\int H_{i}(x) d x=\frac{\left(\left(x-c_{i}\right)^{2}+a_{i}^{2}\right)^{3 / 2}}{6} \\
& +\frac{a_{i}^{2}}{2}\left(x-c_{i}\right) \ln \left(\left(x-c_{i}\right)+\left(\left(x-c_{i}\right)^{2}+a_{i}^{2}\right)^{1 / 2}\right) \\
- & \frac{a_{i}^{2}}{2}\left(\left(x-c_{i}\right)^{2}+a_{i}^{2}\right)^{1 / 2}
\end{aligned}
$$

in which $\left\{c_{i}\right\}_{i=1}^{m}$ is a set of centres and $\left\{a_{i}\right\}_{i=1}^{m}$ is a set of RBF widths.

A set of collocation points $\left\{x_{i}\right\}_{i=1}^{m}$ is taken to be a set of centres, while the RBF width is chosen according to the following relation

$$
a_{i}=\beta d_{i},
$$

where $\beta$ is a factor and $d_{i}$ is the minimum distance from the $i$ th centre to its neighbouring centres. A factor $\beta$ is simply chosen to be unity in the present study. Since $C_{1}$ and $C_{2}$ are to be found, we treat them like the RBF weights.

\subsection{Incorporation IRBFNs into the multiscale basis function framework}

The problem domain is represented using a set of $N$ nodal points, called a global coarse-scale grid. This grid is used for solving the coarse-scale equation (1). On each interval or coarse element, $\left[x_{i-1}, x_{i}\right]$ with $i=\{2,3, \ldots, N\}$, an approximation to the field variable $u$ is sought in the form

$$
u(x)=\phi_{i-1}(x) u_{i-1}+\phi_{i}(x) u_{i},
$$

where $x_{i-1} \leq x \leq x_{i}, u_{i-1}=u\left(x_{i-1}\right), u_{i}=u\left(x_{i}\right)$, and $\phi_{i-1}(x)$ and $\phi_{i}(x)$ the basis functions.

We employ subregion collocation to discretise (1). Each node $x_{i}$ with $i=$ $\{2,3, \ldots, N-1\}$ is surrounded by a control volume $\left[x_{i-1 / 2}, x_{i+1 / 2}\right]$ denoted by $\Omega_{i}$ (Figure 1 ). Integrating (1) over a control volume $\Omega_{i}$, one has

$$
a^{\varepsilon}\left(x_{i+1 / 2}\right) \frac{d u}{d x}\left(x_{i+1 / 2}\right)-a^{\varepsilon}\left(x_{i-1 / 2}\right) \frac{d u}{d x}\left(x_{i-1 / 2}\right)+\int_{x_{i-1 / 2}}^{x_{i+1 / 2}} f d x=0
$$

Taking (8) into account, one can express first derivatives in (9) in terms of nodal values of $u$. Opposed to traditional discretisation methods, the basis functions $\phi_{i-1}(x)$ and $\phi_{i}(x)$ on a coarse element $\left[x_{i-1}, x_{i}\right]$ are not analytical functions (e.g. not polynomials), but local numerical solutions to the following differential 


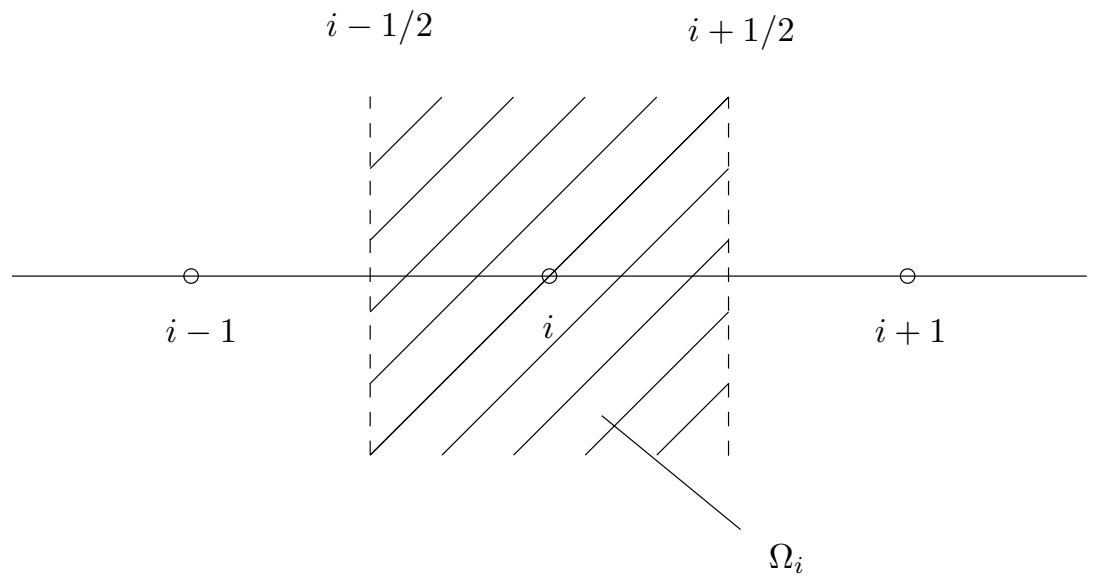

Figure 1: A CV discretisation scheme: node $i$ and its associated control volume. The circles represent the nodes, and the vertical dash lines represent the faces of the control volume.

equation

$$
\frac{d}{d x}\left(a^{\varepsilon} \frac{d \phi_{k}}{d x}\right)=0
$$

with $k=\{i-1, i\}$ and $x_{i-1} \leq x \leq x_{i}$. Boundary conditions for (10) are specified using the condition $\phi_{k}\left(x_{j}\right)=\delta_{k j}$ with $j=\{i-1, i\}$. Equation (10) needs be solved twice for the determination of the two $\phi_{k}$ on each element. A coarse element $\left[x_{i-1}, x_{i}\right]$ is discretised by a set of $M$ points, called a local fine-scale grid. Such a grid is used to capture the fine-scale structure information of the solution. Let $\left\{\eta_{1}=x_{i-1}, \eta_{2}, \ldots, \eta_{M}=x_{i}\right\}$ be a set of nodes of the local fine-scale grid. Collocation of (10) at a set of grid points together with imposition of Dirichlet boundary conditions at the two end points result in the following set of algebraic equations

$$
\mathcal{A} \widehat{w}=\widehat{b}
$$

where

$$
\mathcal{A}=\left[\begin{array}{cccccc}
G_{1}\left(\eta_{1}\right), & G_{2}\left(\eta_{1}\right), & \ldots, & G_{M}\left(\eta_{1}\right), & 0, & 0 \\
G_{1}\left(\eta_{2}\right), & G_{2}\left(\eta_{2}\right), & \ldots, & G_{M}\left(\eta_{2}\right), & 0, & 0 \\
\vdots & \vdots & \ddots & \vdots & \vdots & \vdots \\
G_{1}\left(\eta_{M}\right), & G_{2}\left(\eta_{M}\right), & \ldots, & G_{M}\left(\eta_{M}\right), & 0, & 0 \\
\bar{H}_{1}\left(\eta_{1}\right), & \bar{H}_{2}\left(\eta_{1}\right), & \ldots, & \bar{H}_{M}\left(\eta_{1}\right), & \eta_{1}, & 1 \\
\bar{H}_{1}\left(\eta_{M}\right), & \bar{H}_{2}\left(\eta_{M}\right), & \ldots, & \bar{H}_{M}\left(\eta_{M}\right), & \eta_{M}, & 1
\end{array}\right]
$$


246 Boundary Elements and Other Mesh Reduction Methods XXXIII

$$
\widehat{b}=\left(0,0, \ldots, 0, \phi_{k}\left(x_{i-1}\right), \phi_{k}\left(x_{i}\right)\right)^{T}
$$

It can be seen from (11) that, owing to the presence of the two integration constants, equation (10) is forced to be satisfied exactly not only at the interior points but also at the two end points.

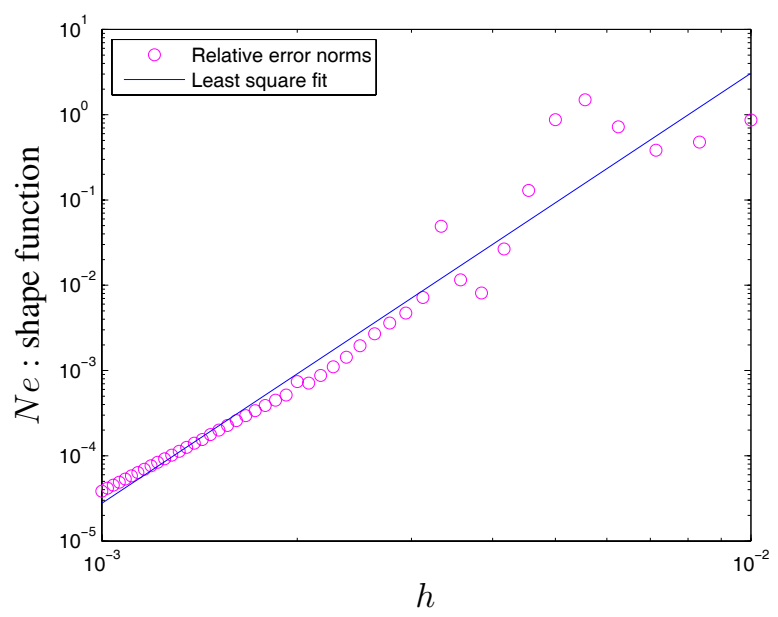

Figure 2: Fine scale convergence.

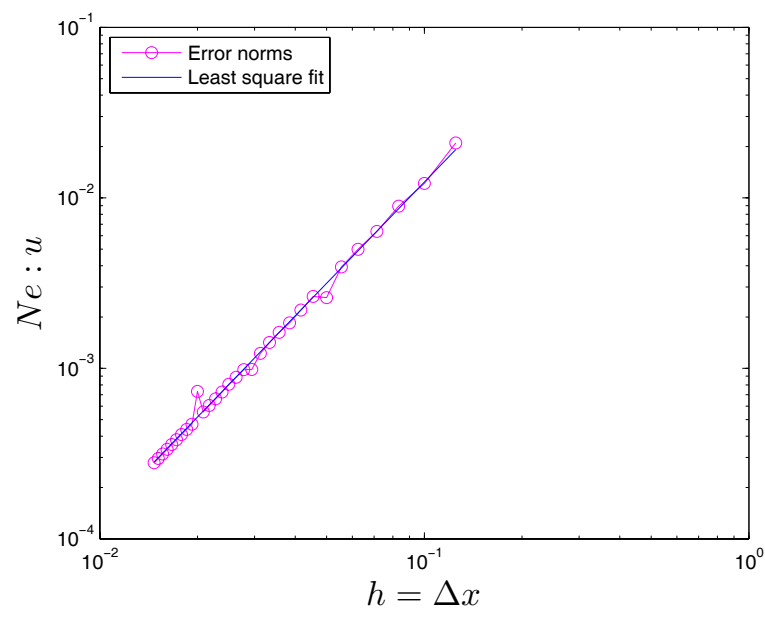

Figure 3: Coarse scale convergence. 


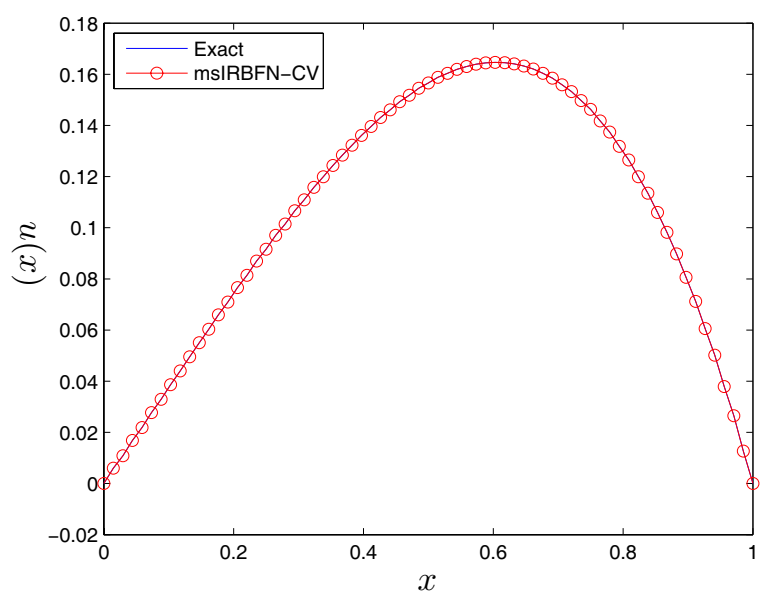

Figure 4: msIRBFN-CV solution.

\section{Numerical results}

Values of $\phi_{k}, d \phi_{k} / d x$ and $d^{2} \phi_{k} / d x^{2}$ at an arbitrary point $x \in\left[x_{i-1}, x_{i}\right]$ can then be computed by

$$
\begin{aligned}
\phi_{k}(x) & =\left[\bar{H}_{1}(x), \bar{H}_{2}(x), \ldots, \bar{H}_{M}(x), x, 1\right] \mathcal{A}^{-1} \widehat{b}, \\
\frac{d \phi_{k}}{d x}(x) & =\left[H_{1}(x), H_{2}(x), \ldots, H_{M}(x), 1,0\right] \mathcal{A}^{-1} \widehat{b}, \\
\frac{d^{2} \phi_{k}}{d x^{2}}(x) & =\left[G_{1}(x), G_{2}(x), \ldots, G_{M}(x), 0,0\right] \mathcal{A}^{-1} \widehat{b} .
\end{aligned}
$$

Figures 2-9 summarise our multiscale modelling of $d\left(a^{\varepsilon}(x) d u(x) / d x\right)+$ $f(x)=0$ where $f(x)=x, a^{\varepsilon}(x)=1 /(2+x+\sin (2 \pi x / \varepsilon))$ and $\varepsilon=0.01$. The domain is discretised using a series of $8,10, \ldots, 68$ uniform coarse elements (i.e. $N=\{9,11, \ldots, 69\})$ and the shape functions that capture the fine scale physics in each coarse element are numerically obtained with our IRBFN collocation method using 55 uniform nodes (i.e. $M=55$ ).

Figure 2 displays the behaviour of the numerical shape functions obtained by our IRBFN collocation method on the interval $0 \leq x \leq 0.1$ with a relative $L_{2}$ error norm of $N e=3.8451 \times 10^{-5}$ and a convergence rate of 5.0434. The coarse scale solution at the coarse grid points is obtained by a conservative control volume method. In order to have a good and consistent measure of accuracy, error norms in all cases are computed using the same 10,000 test points where the fine-scale solution is recovered via simple multiplication of the coarse results with the shape functions at the test points. 
248 Boundary Elements and Other Mesh Reduction Methods XXXIII

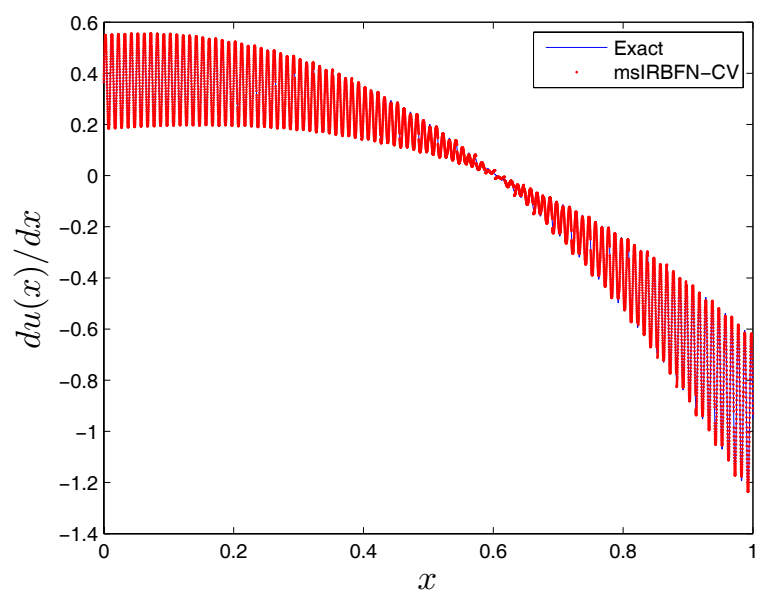

Figure 5: Fine scale recovery: $d u(x) / d x$.

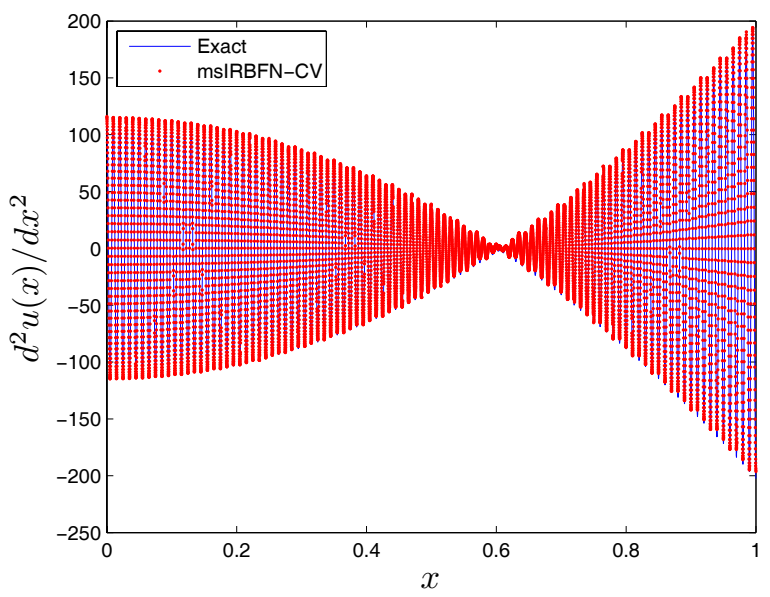

Figure 6: Fine scale recovery: $d^{2} u(x) / d x^{2}$.

Figure 3 shows that our msIRBFN-CV approach achieves a convergence rate of 1.9712 and a relative $L_{2}$ error norm of $N e=2.7942 \times 10^{-4}$ (69 coarse nodes) for this problem.

Figure 4 shows the coarse scale solution.

Figures 5 and 6 display the recovered fine scale results for the first and second derivatives of $u(x)$.

Figures 7,8 and 9 display the results on a small sub-domain $0 \leq x \leq 0.1$ in order to show the fine-scale details better. 


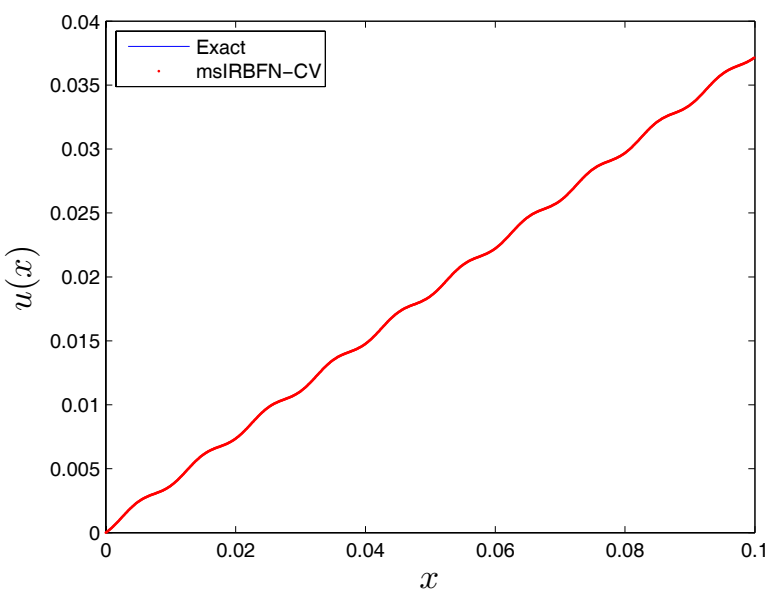

Figure 7: Fine scale: $u(x), 0 \leq x \leq 0.1$.

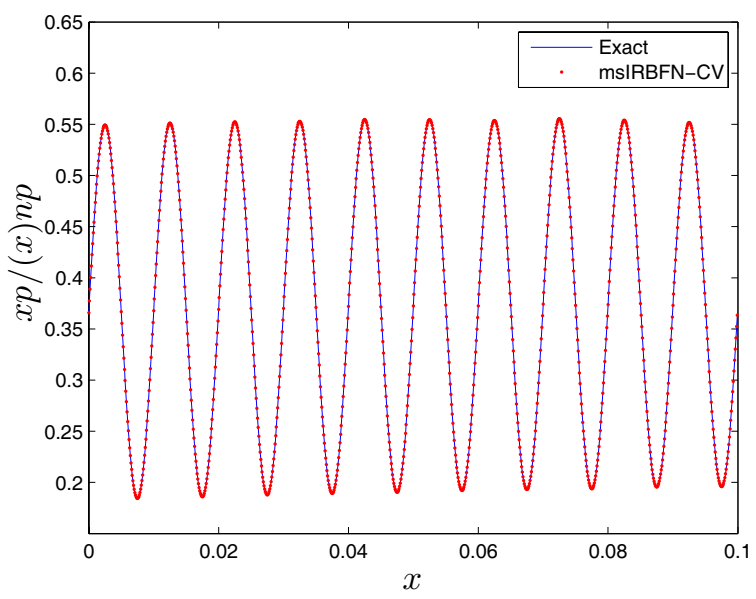

Figure 8: Fine scale: $d u(x) / d x$.

\section{Concluding remarks}

In this paper, we have successfully introduced IRBFNs into the multiscale basis function framework. High rates of convergence for the solution of the fine scale equation are obtained. Numerical results show that our msIRBFN-CV approach captures the fine details quite well. However, we can only guarantee a $C^{0}$ solution. We are currently investigating ways to improve the choice of shape functions. 


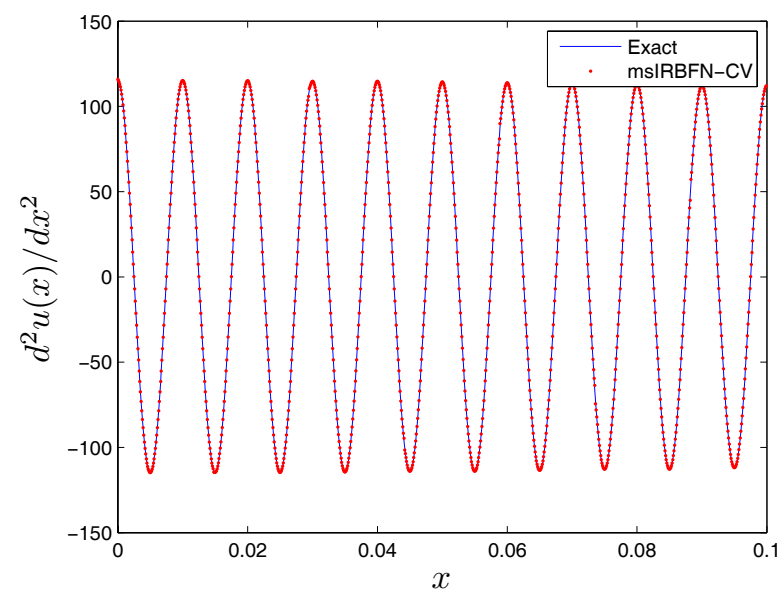

Figure 9: Fine scale: $d^{2} u(x) / d x^{2}$.

\section{Acknowledgement}

This work is supported by the Australian Research Council through a Discovery Projects grant.

\section{References}

[1] Dykaar, B.B. \& Kitanidis, P.K., Determination of the effective hydraulic conductivity for heterogeneous porous media using a numerical spectral approach 1. Method. Water Resources Research, 28, pp. 1155-1166, 1992.

[2] McCarthy, J.F., Comparison of fast algorithms for estimating large-scale permeabilities of heterogeneous media. Transport in Porous Media, 19, pp. 123-137, 1995.

[3] Hou, T.Y. \& Wu, X.-H., A multiscale finite element method for elliptic problems in composite materials and porous media. J. of Computational Physics, 134, pp. 169-189, 1997.

[4] Babuška, I., Caloz, G., \& Osborn, J.E., Special finite element methods for a class of second order elliptic problems with rough coefficients. SIAM J. on Numerical Analysis, 31, pp. 945-981, 1994.

[5] Allaire, G. \& Brizzi, R., A multiscale finite element method for numerical homogenization. Multiscale Modeling \& Simulation, 43, pp. 790-812, 2005.

[6] Hou, T.Y., Multiscale modelling and computation of fluid flow. Int. J. for Numerical Methods in Fluids, 47, pp. 707-719, 2005.

[7] Aarnes, J.E., Kippe, V. \& Lie, K.-A., Mixed multiscale finite elements and streamline methods for reservoir simulation of large geomodels. Advances in Water Resources, 28, pp. 257-271, 2005. 
[8] Arbogast, T., Implementation of a locally conservative numerical subgrid upscaling scheme for two-phase Darcy flow. Computational Geosciences, 6 , pp. 453-481, 2002.

[9] Chu, J., Efendiev, Y., Ginting, V. \& Hou, T.Y., Flow based oversampling technique for multiscale finite element methods. Advances in Water Resources, 31, pp. 599-608, 2008.

[10] Hajibeygi, H., Bonfigli, G., Hesse, M.A. \& Jenny, P., Iterative multiscale finite-volume method. J. of Computational Physics, 227, pp. 8604-8621, 2008.

[11] Hou, T.Y., Wu, X.-H. \& Cai, Z., Convergence of a multiscale finite element method for elliptic problems with rapidly oscillating coefficients. Mathematics of Computation, 68, pp. 913-943, 1999.

[12] Fasshauer, G.E., Meshfree Approximation Methods With Matlab (Interdisciplinary Mathematical Sciences - Vol. 6), World Scientific Publishers: Singapore, 2007.

[13] Mai-Duy, N. \& Tran-Cong, T., Numerical solution of differential equations using multiquadric radial basis function networks. Neural Networks, 14, pp. 185-199, 2001.

[14] Mai-Duy, N. \& Tran-Cong, T., Numerical solution of Navier-Stokes equations using multiquadric radial basis function networks. Int. J. for Numerical Methods in Fluids, 37, pp. 65-86, 2001. 